\title{
Iatrogenic nasal synechiae in a premature newborn
}

\author{
Alper Aykanat ${ }^{1 \oplus}$, Hasan Tolga Çelik $^{1 \oplus}$, Rıza Önder Günaydın $^{2 \odot}$, Şule Yiğit ${ }^{1 \odot}$ \\ ${ }^{1}$ Division of Neonatology, Department of Pediatrics and ${ }^{2}$ Department of Otorhinolaryngology, Hacettepe University Faculty of \\ Medicine, Ankara, Turkey.
}

\begin{abstract}
Background. Nasal obstruction may cause short- and long-term problems such as respiratory distress, cyanosis, apnea, difficulty during feeding, and failure to thrive during the newborn period; since newborns are obligatory nasal breathers. Compression effect of the nasal cannulas and prongs used during respiratory support and nasal aspiration applications to clear the airways of secretions may result in nasal synechiae and acquired iatrogenic nasal obstruction.
\end{abstract}

Case. In this case report, we present a premature newborn with nasal synechiae secondary to long-term nasal continuous positive airway pressure (nasal CPAP) applications and routine upper airway nursing care.

Conclusion. Severe nasal damage may occur in premature newborns receiving prolonged nasal CPAP support. To prevent this upper airway care should be conducted as gently as possible in premature newborns.

Key words: neonatology, nasal obstruction.

Newborn babies are obligatory nasal breathers. Short- and long-term problems such as respiratory distress, cyanosis, apnea, difficulty during feeding, and failure to thrive may be seen in patients with nasal obstruction..$^{1-3}$ Etiology of nasal obstruction in neonates involve congenital and acquired causes. Congenital anomalies include nasal agenesis, choanal stenosis/atresia, craniofacial anomalies, septal deviations and congenital masses. Acquired causes include obstructions due to birth traumas, infectious rhinitis, scar formation due to compression effect of nasal cannulas and prongs used during respiratory support, and nasal synechiae secondary to nasal aspirations to clear secretions. ${ }^{1-5}$ In this case report, a premature newborn with nasal synechiae secondary to long-term nasal continuous positive airway pressure (nasal CPAP) applications and routine upper airway nursing care is presented.

\footnotetext{
$\bowtie$ Alper Aykanat

alper5334@windowslive.com
}

Received 22nd August 2019, revised 23rd September, accepted 27th September 2019.

\section{Case Report}

A female neonate weighing 1160 grams (1050p) was born via emergent cesarean section as the second baby from a twin-gestation of $30+5$ weeks to a 40-year-old primipara mother because of clinically confirmed acute appendicitis in the mother and documented decelerations upon non-stress test examination. The baby was intubated and admitted to the newborn intensive care unit.

Prenatal history of the mother revealed primary hypertension, type 2 diabetes mellitus regulated with insulin therapy, Familial Mediterranean Fever regulated with colchicine therapy, sickle cell anemia trait, gluten-induced enteropathy and multinodular goiter. Fetal DNA analysis and antenatal ultrasonographic examinations were normal. There was no consanguinity between mother and father.

Starting from first days of life, thin nasal suction catheters did not progress easily through both nasal passages. Because bilateral choanal patency was revealed immediately after birth, findings were evaluated as an acquired stenosis, and nasal examination was planned. 
Three doses of intratracheal surfactant therapy were introduced within the first 48 hours of life. Grade 3 intraventricular hemorrhage was detected during routine cranial ultrasonography on the 3rd day of life. Surgical ligation was performed due to hemodynamically significant and pharmacologically non-reactive patent ductus arteriosus on 25th day of life. Intubated mechanical ventilation was introduced for a total of 27 days. Extubation was successful on 27 th day of life, and respiratory support was continued as nasal CPAP for a total of 35 days. This support was interchanged with nasal masks and bi-nasal prongs. On 35th and 58th days of life, two courses of dexamethasone therapy $(0.5 \mathrm{mg} / \mathrm{kg} /$ day, 3 days) were given for bronchopulmonary dysplasia. Vitamin A treatment (5000 IU/dose, 3 doses/week, orally) was given starting from 35th day of life for a total of 3 weeks. Appropriate continuous moistening of the airway with humidifiers was introduced throughout the respiratory support at all times. Secretions were effectively cleared from the upper airways whenever needed with
6-Fr nasal suction catheters, with a negative pressure less than $80 \mathrm{~mm} \mathrm{Hg}$.

Due to lack of separation from Nasal CPAP, oxygen dependency ( $\mathrm{FiO} 2$ 50\%) during feeding sessions, and increasingly difficult progression of nasal suction catheters during nasal aspirations, the patient was evaluated by otorhinolaryngology team on 60th day of life. Flexible fiberoptic nasal endoscopy revealed bilateral intensive nasal synechiae that occluded bilateral nasal passages almost totally. (Fig. 1) To prevent potential subsequent scars and synechiae, no endoscopic surgical intervention was performed. Palliative interventions to relieve the symptoms were introduced to the patient, such as serum physiologic nasal drops and intranasal steroids. Nasal aspirations were discontinued.

On 67th day of life, the patient was spontaneously separated from Nasal CPAP but continued to receive free oxygen. Gastrostomy procedure was planned for the patient, who had unsuccessful oral feeding sessions due
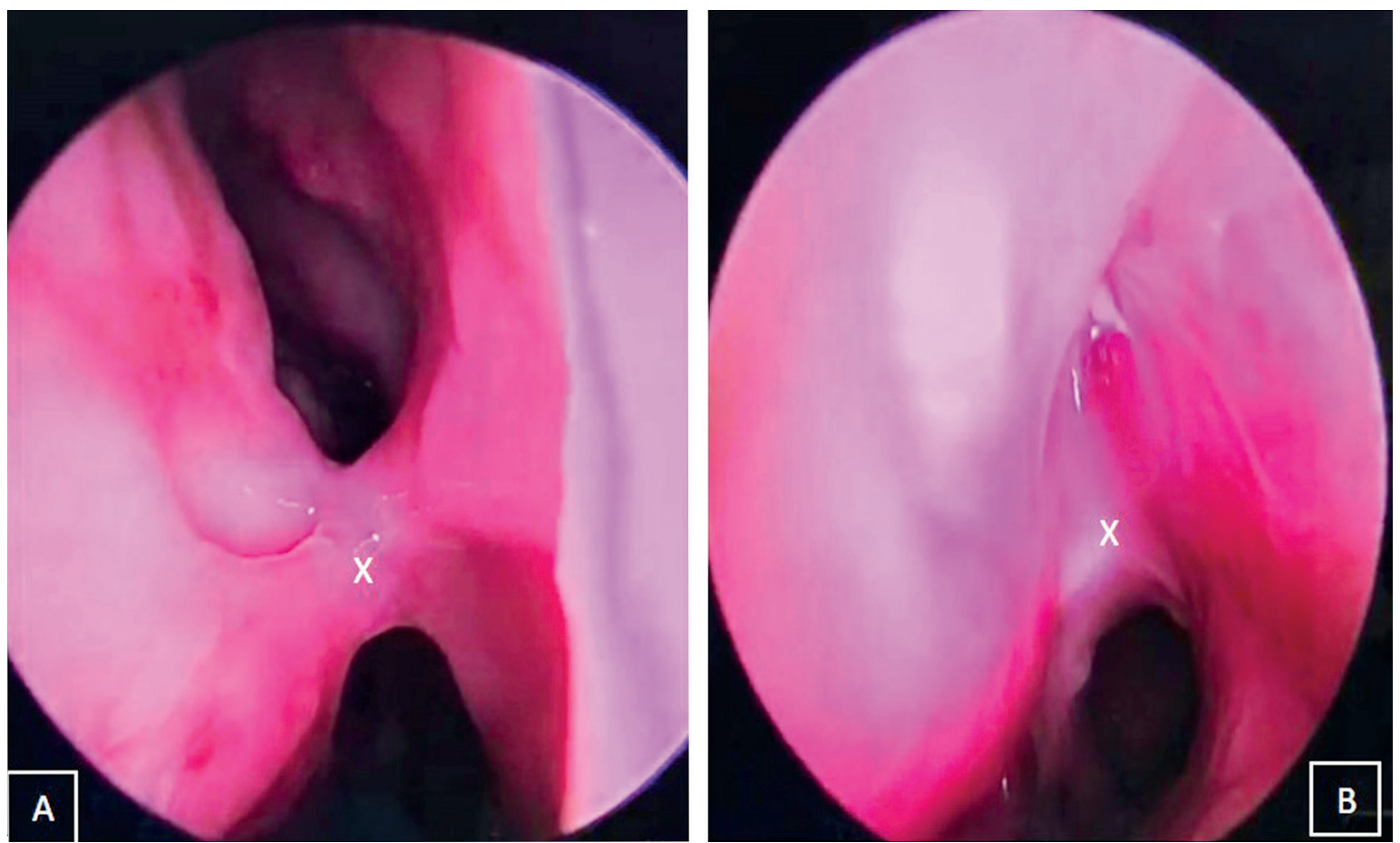

Fig. 1. Nasal cavity examination under Fiberoptic Flexible Bronchoscopy. A. Right nasal cavity. B. Left nasal cavity. X: Areas of synechiae. 
to nasal passage narrowing and could only be fed with the help of orogastric feeding catheters. During the 20 days of pre-operative period, the patient received only free oxygen, bilateral nasal passages began to relieve, oxygen dependency gradually decreased and ultimately disappeared. Oral feeding was successful on 90th day of life. Reevaluation with the help of flexible fiberoptic nasal endoscopy revealed that nasal passage at the right choana was open and much improved. Patient was discharged on 99th day of life with outpatient follow-up planning. Informed consent has been obtained from the mother of the baby.

\section{Discussion}

Nasal obstructions may lead to short- and longterm morbidities such as respiratory distress, cyanosis, apnea, difficulty during feeding, and failure to thrive in newborns. Narrowing of nasal passages due to acquired causes is usually a manifestation of iatrogenic nasal trauma caused by nasal aspirations and results in progressively increasing respiratory distress and unsuccessful weaning from respiratory support. ${ }^{1-5}$

At present, the preferred method of noninvasive respiratory support in newborns is nasal CPAP application. To minimize pressure leakage, well-sitting interfaces are used. These interfaces include triangular mask-shaped soft interfaces that are closed on the nose and bi-nasal soft short prongs in the form of short tubes that fit on both nostrils. Interchanging use of these interfaces is recommended to minimize microtraumas. ${ }^{1}$

Regular cleaning of upper airways from secretions to keep passages open is especially needed in premature babies because their airways are relatively narrower and have a high resistance to air flow, which results in considerable decrease in delivery of nasal CPAP pressure to lungs when airways are obstructed by secretions. Because nasal aspirations itself may result in iatrogenic tissue damage, it should not be performed when patient has no need, should preferably be performed as gently as possible and should be performed with a negative pressure less than $80 \mathrm{mmHg}$. ${ }^{1}$

In the literature, wide spectrum of complications due to Nasal CPAP and upper airway aspirations were reported. ${ }^{4,5}$ One study reported that nasal synechiae developed as a complication of nasal CPAP treatment in two (82\%) of 82 premature newborns. In one of the babies' synechiae were bilateral, and was sent home with nasal oxygen at discharge. Because of nasal oxygen dependency, balloon dilatation was performed on the nasal cavity, but synechiae continued to be present at one-year follow-up. Synechiae were unilateral in the other infant, who had no respiratory problems and no feeding difficulties and thus needed no surgical intervention. ${ }^{4}$

In another study, which included 91 premature newborn babies who all received nasal CPAP, intranasal ulcerations were detected in six $(3.3 \%)$ nostrils, granulations in nasal cavity were present in three $(1.6 \%)$ nostrils, four nostrils $(2.2 \%)$ had stenosis of the vestibular region. ${ }^{5}$

In our case, the nasal passages, which were already narrow, became progressively and nearcompletelyobstructed secondary tomicrotrauma caused by nasal aspirations and nasogastric feeding catheters. Respiratory distress and feeding difficulty lasted long enough to suggest a gastrostomy procedure. Enlargement of nasal passages with advancement of postnatal age and discontinuation of nasal aspirations resulted in relief of nasal passages and unilateral re-opening of nasal airway. Cessation of respiratory distress made it possible to feed the infant orally. Because nasal synechiae are secondary to inflammatory response caused by microtraumas in nasal passages, as in the case presented here, aim should be to eliminate main causes of microtraumas as well as to relieve the symptoms of patient palliatively, rather than directly intervening with surgical procedures.

In conclusion, severe nasal damage may occur in premature newborns who receive nasal 
CPAP support for long periods of time. Ongoing respiratory distress and unsuccessful weaning from respiratory support negatively affect oral feeding and weight gain, which results in prolonged hospitalization. Upper airway care should be done as gently as possible in premature newborns. Upper airway examinations should be done whenever needed to diagnose potential obstructions and synechiae.

\section{REFERENCES}

1. Yurdakök M (ed). Mekanik ventilasyon. İçinde: Yurdakök M, Yiğit Ş, Çelik HT (ed).Yenidoğan Bakımında Hacettepe Uygulamaları (3. Basım). Ankara: Güneş Tıp Kitapevleri, 2019: 86-108.
2. Roehm CE, Lawrason A, Valdez TA. Nasal obstruction in newborn. In: Kountakis SE (ed). Encyclopedia of Otolaryngology, Head and Neck Surgery. Berlin, Heidelberg: Springer, 2013: 17461757.

3. Yong SC, Chen SJ, Boo NY. Incidence of nasal trauma associated with nasal prong versus nasal mask during continuous positive airway pressure treatment in very low birthweight infants: a randomised control study. Arch Dis Child Fetal Neonatal Ed 2005; 90: F480-F483.

4. Gökdoğan O, İleri F. Nasal complications related with cpap treatment. ENT Updates 2018; 8: 133-138.

5. Jatana KR, Oplatek A, Stein M, Phillips G, Kang DR, Elmaraghy CA. Effects of nasal continuous positive airway pressure and cannula use in the neonatal intensive care unit setting. Arch Otolaryngol Head Neck Surg 2010; 136: 287-291. 